\title{
The burden of oncology promises not kept in glioblastoma
}

\author{
Giuseppe Lamberti ${ }^{1}$, Enrico Franceschi ${ }^{1}$ \& Alba A Brandes*, 1 \\ ${ }^{1}$ Department of Medical Oncology, Azienda USL, Bellaria Hospital - IRCCS Institute of Neurological Sciences, Bologna, Italy \\ * Author for correspondence: alba.brandes@yahoo.it
}

\begin{abstract}
"New drugs and therapeutic approaches have been evaluated in Phase III trials but none have showed a survival improvement in newly diagnosed or recurrent glioblastoma in the last 10 years. Nevertheless, refinement of clinical management over the years has led to an increase in OS"
\end{abstract}

First draft submitted: 18 September 2017; Accepted for publication: 25 October 2017; Published online: 17 January 2018

Keywords: CheckMate • clinical trial • glioblastoma • immunotherapy • ipilimumab • nivolumab

Glioblastoma is the most common primary CNS tumor and is the most aggressive neoplasm among gliomas. Glioblastoma represents a highly unmet medical need due to its dismal prognosis, meaningful disability and lack of new agents since temozolomide concurrent with and adjuvant to radiotherapy became the standard therapy [1].

\section{Immunotherapy clinical trials}

In recent years, a renewed interest has been raised in immunotherapy of solid tumors following remarkable results in trials on difficult-to-treat neoplasms such as melanoma and non-small-cell lung cancer. The breakthrough for this immunotherapy takeover came through the targeting of immune checkpoint proteins. The first compounds proven to be effective were in fact directed against the CTLA-4 and PD-1. CTLA-4 on the membrane of T-lymphocytes binds its costimulatory ligand B7/CD28 expressed by antigen-presenting cells and inhibits lymphocyte activation. Similarly, PD-1 is a membrane receptor expressed on activated T-lymphocytes that induces anergy upon binding the PD-L1 ligand on tumor cells. The immune checkpoint CTLA-4 is targeted by ipilimumab, whereas PD-1 is targeted by nivolumab and pembrolizumab and PD-L1 by atezolizumab and avelumab, respectively. These drugs interfere with the immune escape mechanisms adopted by tumor cells to induce host immune system tolerance [2].

Ipilimumab, a human monoclonal IgG1 antibody anti-CTLA-4, has been shown to significantly prolong survival in patients affected by advanced melanoma, resulting in its approval as first-line treatment. Immune-related adverse events were a concern, since toxic deaths occurred in patients treated with ipilimumab, most of them due to colitis [3]. Physicians, however, became more confident with the drug and management of adverse events and earlier administration of steroids improved patients' outcome, so that drug-related deaths became exceptional in tertiary centers [4].

Nivolumab is a human monoclonal IgG4 antibody against PD-1, which is proven to be effective and has been approved for the treatment of various cancers, including melanoma, non-small-cell lung cancer, head and neck cancers, renal cell carcinoma, urothelial carcinoma and Hodgkin's lymphoma. The toxicity profile of nivolumab is more favorable compared with ipilimumab and adverse events are less common and milder in severity than those induced by the anti-CTLA-4 antibody.

\section{Immunotherapy for glioblastoma}

The hope of new therapeutic options in glioblastoma patients has been raised by survival rates reported from previous immunotherapy studies and data on potential activity across the blood-brain barrier [5]. However, a recent report on 16 glioblastoma patients receiving nivolumab upon progression to bevacizumab showed no survival advantage, with 2 months progression free and 3.5 months survival [6]. Similarly, 17 adult patients with progressive brain tumor treated with pembrolizumab, showed median survival of 2.6 months [7]. 
The CheckMate-143 study (NCT02017717) was designed with Phase I and III parts to explore the role of nivolumab and ipilimumab in recurrent glioblastoma [8]. In safety cohorts, patients received monotherapy with nivolumab $3 \mathrm{mg} / \mathrm{kg}$ every 2 weeks or four doses of nivolumab $1 \mathrm{mg} / \mathrm{kg}$ plus ipilimumab $3 \mathrm{mg} / \mathrm{kg}$ every 3 weeks followed by nivolumab $3 \mathrm{mg} / \mathrm{kg}$ every 2 weeks or four doses of nivolumab $3 \mathrm{mg} / \mathrm{kg}$ plus ipilimumab $1 \mathrm{mg} / \mathrm{kg}$ every 3 weeks followed by nivolumab $3 \mathrm{mg} / \mathrm{kg}$ every 2 weeks. $9 / 10$ and 14/20 patients experienced grade 3-4 adverse events in the nivolumab $1 \mathrm{mg} / \mathrm{kg}$ plus ipilimumab $3 \mathrm{mg} / \mathrm{kg}$ combination cohort and in the nivolumab $3 \mathrm{mg} / \mathrm{kg}$ plus ipilimumab $1 \mathrm{mg} / \mathrm{kg}$ combination cohort, respectively. Nivolumab monotherapy was well tolerated: as recently presented at the ESMO 2017 Congress, grade 3-4 adverse event rate was 33-41\% leading to treatment discontinuation in $8-10 \%$ of patients [9].

Given the encouraging $40 \%$ overall survival (OS) rate at 12 months in the nivolumab cohort, the Phase III cohort enrollment (nivolumab at $3 \mathrm{mg} / \mathrm{kg}$ every 2 weeks vs bevacizumab $10 \mathrm{mg} / \mathrm{kg}$ every 2 weeks) started in 2014 . Nevertheless, the trial was declared to be negative earlier this year at the World Federation of Neuro-Oncology Societies as the nivolumab arm did not meet the primary end point of improved OS when compared with bevacizumab arm. However, a long median duration of response (11 months) was seen in patients responding to nivolumab (8\%) [10].

In newly diagnosed glioblastoma, results from two clinical trials on nivolumab, the CheckMate-498 and the CheckMate-548, are awaited. The CheckMate-498 trial (NCT02617589) is a randomized Phase III open-label study with radiation therapy combined with either nivolumab ( $240 \mathrm{mg}$ every 2 weeks for 16 weeks and then $480 \mathrm{mg}$ every 4 weeks) or temozolomide in newly diagnosed MGMT unmethylated glioblastoma. The CheckMate-548 trial (NCT02667587) is a randomized Phase III study on radiotherapy plus temozolomide combined with nivolumab ( 240 mg every 2 weeks for 16 weeks and then 480 mg every 4 weeks) or placebo in newly diagnosed methylated MGMT glioblastoma.

\section{Vaccination-based approaches}

Targeting immune checkpoints was not the only strategy planned to induce host immune response against tumor cells. Vaccination is a widely explored approach, requiring a tumor-associated neoantigen to be identified.

A truncated variant of the EGFR characterized by loss of exons 2-7 is expressed in around $20 \%$ of glioblastomas and is referred to as EGFRvIII [11]. Rindopepimut or CDX-110, is a vaccine made up of a peptide conjugated to the carrier protein keyhole limpet hemocyanin, developed to induce immune response against glioblastoma cells expressing EGFRvIII. In the Phase III trial (ACT IV), patients with newly diagnosed glioblastoma were randomized to receive rindopepimut $(\mathrm{n}=371)$ or control with keyhole limpet hemocyanin $(\mathrm{n}=374)$ together with standard adjuvant temozolomide after surgical resection and completion of radiotherapy and concomitant temozolomide. Rindopepimut failed to improve OS, which was the primary end point of the study, when compared with control $\operatorname{arm}(20.1$ vs 20.0 months) [12]. Although the ACT IV is formally a negative study, rindopepimut may need further evaluation given the better-than-expected performance of the control group and the possibility that standard-dose temozolomide may have induced lymphocytopenia and immunosuppression in experimental arm [13]. The target population that is more likely to benefit from rindopepimut therapy may need to be better defined, possibly by optimization of selection criteria for residual disease after surgery or technique and cutoff used for definition of EGFRvIII expression versus nonexpression.

\section{Molecular targeting}

Another interesting field is molecular targeting. Efforts are made to exploit IDH-1 and -2 mutations. These seem to be promising druggable targets in secondary glioblastoma, in other words, glioblastoma progressing from lower grade lesions. Phase I trials on IDH1-2 inhibitors are ongoing, such as on AG-881 (NCT02481154) or on CB839 (NCT02071862). In addition, inhibition of nicotinamide phosphoribosyltransferase (NAMPT) could have a promising role since NAD+ depletion has been found to be a metabolic vulnerability of IDH-1 mutant cancers [14]. At last, safety and activity of peptide vaccines designed against the IDH1 R132H mutation are under investigation in early clinical trials: RESIST (NCT02193347) and NOA-16 (NCT02454634).

\section{Combination therapies}

Additional strategies may improve activity and efficacy of currently available treatments. Combinations of two immune checkpoint inhibitors, such as ipilimumab and nivolumab, are already under investigation in glioblastoma in the safety cohorts of the CheckMate-143 trial. The combination of ipilimumab and nivolumab has improved 
progression-free survival in untreated melanoma patients [15] and showed activity in recurrent small-cell lung cancer and advanced non-small-cell lung cancer [16]. The cost for improved outcomes was the increased adverse events rate as compared with single agent treatment. Future options may also include combination of immune checkpoint inhibitors and vaccines in order to overcome tumor-induced immunosuppression and enhance activity, following recent preclinical data [17]. Indoleamine 2,3-dioxygenase (IDO) is another checkpoint molecule expressed by glioblastoma to suppress immune function [18]. Preclinical models showed that strategies involving IDO inhibition were active [19,20], in fact, Phase I clinical trials with indoximod, an IDO inhibitor started (NCT02052648).

\section{Conclusion}

Glioblastoma treatment remains challenging. New drugs and therapeutic approaches have been evaluated in Phase III trials but none have showed a survival improvement in newly diagnosed or recurrent glioblastoma in the last 10 years. Nevertheless, refinement of clinical management over the years has led to an increase in OS, as seen in the control group of the ACT IV study [12].

The breakthrough of immune oncology is still awaited by glioblastoma patients and oncologists. Improvement in safety and tolerability will have a key role for such therapies to take over in glioblastoma patients, as well as combinations of promising approaches.

\section{Financial \& competing interests disclosure}

The authors have no relevant affiliations or financial involvement with any organization or entity with a financial interest in or financial conflict with the subject matter or materials discussed in the manuscript. This includes employment, consultancies, honoraria, stock ownership or options, expert testimony, grants or patents received or pending, or royalties.

No writing assistance was utilized in the production of this manuscript.

\section{References}

1 Stupp R, Mason WP, van den Bent MJ et al. Radiotherapy plus concomitant and adjuvant temozolomide for glioblastoma. N. Engl. J. Med. 352(10), 987-996 (2005).

2 Buchbinder EI, Desai A. CTLA-4 and PD-1 pathways: similarities, differences, and implications of their inhibition. Am. J. Clin. Oncol. 39(1), 98-106 (2016).

3 Hodi FS, O’Day SJ, McDermott DF et al. Improved survival with ipilimumab in patients with metastatic melanoma. N. Engl. J. Med. 363(8), 711-723 (2010).

4 O'Connor A, Marples M, Mulatero C, Hamlin J, Ford AC. Ipilimumab-induced colitis: experience from a tertiary referral center. Therap. Adv. Gastroenterol. 9(4), 457-462 (2016).

5 Franceschi E, Minichillo S, Brandes AA. Pharmacotherapy of glioblastoma: established treatments and emerging concepts. CNS Drugs 31(8), 675-684 (2017).

6 Chamberlain MC, Kim BT. Nivolumab for patients with recurrent glioblastoma progressing on bevacizumab: a retrospective case series. J. Neurooncol. 133(3), 561-569 (2017).

7 Blumenthal DT, Yalon M, Vainer GW et al. Pembrolizumab: first experience with recurrent primary central nervous system (CNS) tumors. J. Neurooncol. 129(3), 453-460 (2016).

8 Reardon DA, Sampson JH, Sahebjam S et al. Safety and activity of nivolumab (nivo) monotherapy and nivo in combination with ipilimumab (ipi) in recurrent glioblastoma (GBM): updated results from CheckMate-143. J. Clin. Oncol. 34(15_Suppl.), 2014 (2016).

9 Lim M, Omuro A, Vlahovic G et al. Nivolumab (nivo) in combination with radiotherapy (RT) \pm temozolomide (TMZ): Updated safety results from CheckMate 143 in pts with methylated or unmethylated newly diagnosed glioblastoma (GBM). Ann. Oncol. 28(Suppl. 5), v109 (2017).

10 Reardon DA, Omuro A, Brandes AA et al. Randomized Phase 3 study evaluating the efficacy and safety of nivolumab vs bevacizumab in patients with recurrent glioblastoma: CheckMate 143. Neuro. Oncol. 19(Suppl._3), iii21-iii21 (2017).

11 Weller M, Kaulich K, Hentschel B et al. Assessment and prognostic significance of the epidermal growth factor receptor vIII mutation in glioblastoma patients treated with concurrent and adjuvant temozolomide radiochemotherapy. Int. J. Cancer. 134(10), 2437-2447 (2014).

12 Weller M, Butowski N, Tran DD et al. Rindopepimut with temozolomide for patients with newly diagnosed, EGFRvIII-expressing glioblastoma (ACT IV): a randomised, double-blind, international Phase 3 trial. Lancet Oncol. 18(10), 1373-1385 (2017).

13 Grossman SA, Ye X, Lesser G et al. Immunosuppression in patients with high-grade gliomas treated with radiation and temozolomide. Clin. Cancer Res. 17(16), 5473-5480 (2011).

14 Tateishi K, Wakimoto H, Iafrate AJ et al. Extreme vulnerability of IDH1 mutant cancers to NAD+ depletion. Cancer Cell. 28(6), 773-784 (2015). 
15 Larkin J, Chiarion-Sileni V, Gonzalez R et al. Combined nivolumab and ipilimumab or monotherapy in untreated melanoma. N. Engl. J. Med. 373(1), 23-34 (2015).

16 Hellmann MD, Rizvi NA, Goldman JW et al. Nivolumab plus ipilimumab as first-line treatment for advanced non-small-cell lung cancer (CheckMate 012): results of an open-label, Phase 1, multicohort study. Lancet Oncol. 18(1), 31-41 (2017).

17 Weller M, Roth P, Preusser M et al. Vaccine-based immunotherapeutic approaches to gliomas and beyond. Nat. Rev. Neurol. 13(6), 363-374 (2017).

18 Sordillo PP, Sordillo LA, Helson L. The kynurenine pathway: a primary resistance mechanism in patients with glioblastoma. Anticancer Res. 37(37), 2159-2171 (2017).

19 Wainwright DA, Chang AL, Dey M et al. Durable therapeutic efficacy utilizing combinatorial blockade against IDO, CTLA-4, and PD-L1 in mice with brain tumors. Clin. Cancer Res. 20(20), 5290-5301 (2014).

20 Hanihara M, Kawataki T, Oh-Oka K, Mitsuka K, Nakao A, Kinouchi H. Synergistic antitumor effect with indoleamine 2,3-dioxygenase inhibition and temozolomide in a murine glioma model. J. Neurosurg. 124(6), 1594-1601 (2016). 